\title{
COMPOSIÇÃO FLORÍSTICA DO SUB-BOSQUE DE UMA FLORESTA OMBRÓFILA DENSA MONTANA, MORRETES, PR, BRASIL
}

\author{
Carina Kozera $^{1}$, Ricardo Ribeiro Rodrigues ${ }^{2}$, Vinícius Antonio de Oliveira Dittrich ${ }^{3}$ \\ ${ }^{1}$ Bióloga, Dr ${ }^{\mathrm{a}}$., UFPR, Curitiba, PR, Brasil - carinakozera@ yahoo.com.br \\ ${ }^{2}$ Biólogo, Dr., Escola Superior de Agricultura “Luiz de Queiroz", USP, Piracicaba, SP, Brasil - rrr@ esalq.usp.br \\ ${ }^{3}$ Biólogo, Dr., UESB, Vitória da Conquista, BA, Brasil - vinarc@gmail.com
}

Recebido para publicação: 18/03/2008 - Aceito para publicação: 11/09/2008

\begin{abstract}
Resumo
Foi realizado o levantamento florístico do sub-bosque (estrato inferior) de uma Floresta Ombrófila Densa Montana localizada no Parque Estadual Pico do Marumbi, na encosta do morro Facãozinho, município de Morretes, Paraná. Os objetivos do trabalho foram listar as espécies herbáceas e subarbustivas de angiospermas e de pteridófitas presentes no estrato inferior da floresta e caracterizar a fisionomia do sub-bosque quanto às formas de vida e os principais ambientes de ocorrência das espécies. Para isso, foram realizadas coletas quinzenais, utilizando-se técnicas usuais de coleta e de herborização, e, para a determinação, foram utilizadas bibliografia específica, consultas a especialistas e comparações com exsicatas de herbários. Foram registradas 108 espécies, 68 gêneros e 41 famílias, destacando-se com maior riqueza de espécies Dryopteridaceae (11), Araceae (7), Blechnaceae (6), Piperaceae (6) e Rubiaceae (6). Do total de espécies, cinco foram observadas como rupícolas, oito como rupícolas/terrícolas e 95 como terrícolas. As angiospermas foram representadas por sete formas biológicas e as pteridófitas por quatro. O número de espécies registradas na área foi elevado, quando comparado a outros estudos florísticos realizados com os estratos arbóreo e arbustivo da mesma formação vegetal, ficando demonstranda com isso a importância do componente inferior na diversidade de áreas de Floresta Ombrófila Densa.

Palavras-chave: Estrato inferior; herbácea; sub-bosque; Floresta Ombrófila Densa; Floresta Atlântica.
\end{abstract}

\begin{abstract}
Floristic composition of the understorey of a dense ombrophilous montane forest in Morretes, State of Paraná, Brazil. The floristic composition of the understorey of a Dense Ombrophilous Montane Forest located on a slope of the Morro Facãozinho, Pico do Marumbi State Park, municipality of Morretes, Paraná State, was investigated. The aims of this work were to list of the herbaceous and subshrub species of angiosperms and pteridophytes, and to characterize the physiognomy of the understorey regarding to the life-forms present and the main environments where the different species occur. To achieve this, sampling collections were made twice a month employing usual techniques for collecting and herborizing. Plant identification was carried out using available taxonomic literature. Furthermore, experts were consulted, as well as specimens were compared with exsicattae from some herbaria. One hundred and eight species, 68 genera and 41 families were registered, of which Dryopteridaceae (with 11 species), Araceae (seven), Blechnaceae, Piperaceae, and Rubiaceae (six each) were the richest families. Five out of 108 species were observed as lithophytes, eight as lithophytes or terrestrial plants, and 95 as terrestrial. The flowering plants are represented by seven life-forms, and the pteridophytes by four. The number of species registered is high when compared to that in floristic works carried out on the canopy at the same vegetation type, showing the importance of the understorey in the diversity of areas with dense ombrophilous forest.

Keywords: Understorey; herbs; Dense Ombrophilous Forest; Atlantic Forest.
\end{abstract}

\section{INTRODUÇÃO}

As florestas tropicais da encosta atlântica apresentam variações regionais e locais que as situam entre as florestas mais ricas e diversificadas do território brasileiro (MANTOVANI, 1998). Encontram-se entre as áreas mais importantes de florestas tropicais do mundo e são consideradas prioritárias para a 
realização de levantamentos, principalmente devido ao intenso processo de desmatamento e ao alto índice de endemismos (PRANCE;CAMPBELL, 1988; MORI, 1989).

Estudos têm mostrado uma enorme diversidade no grupo de plantas não-arbóreas presentes nos sub-bosques das florestas tropicais (PRANCE, 1989; ANDREATA et al., 1997). No entanto, as espécies desse grupo frequentemente são negligenciadas, mesmo constituindo parte integrante e com funções específicas na comunidade vegetal da floresta (LASKA, 1997; ANDRADE, 1992; POULSEN; BALSLEV, 1991; SMITH, 1987).

Diferentes autores realizaram estudos buscando explicar as diferentes composições florísticas e/ou estruturais desse componente em diferentes áreas florestais, levando em consideração as exigências específicas das espécies quanto à luminosidade (BERNACCI, 1992; ZICKEL, 1995; POULSEN e PENDRY, 1995; LASKA, 1997), à disponibilidade de água (GENTRY; EMMONS, 1987; ZICKEL, 1995; POULSEN, 1996), aos tipos de solos (GENTRY e EMMONS, 1987; GENTRY, 1988; YOUNG; LÉON, 1989; POULSEN; BALSLEV, 1991; BERNACCI, 1992) e à altitude (REEDER; RIECHERT, 1975; OGDEN; POWELL, 1979; GENTRY, 1988). Pelos resultados apresentados, a distribuição das espécies herbáceas e subarbustivas numa floresta, bem como o crescimento e desenvolvimento dos indivíduos nessas sinúsias estão relacionados a uma interação complexa de todos os fatores citados, resultando em comunidades organizadas nas quais as espécies se relacionam entre si e com o ambiente no qual se encontram presentes.

Para que se possa caracterizar e compreender a dinâmica das florestas tropicais em toda a sua complexidade, são necessários estudos relacionados à composição florística e à estrutura fitossociológica de todos os estratos, inclusive o herbáceo (ANDRADE, 1992). Só dessa forma a determinação e a comparação da riqueza de espécies em diferentes formações vegetais poderá ser estabelecida de forma mais precisa.

Entre os trabalhos realizados especificamente com o componente herbáceo e/ou subarbustivo em formações florestais e que buscaram caracterizá-lo florística e/ou fitossociologicamente, estão o de Citadini-Zanette (1984), Cestaro et al. (1986), Cervi et al. (1987, 1988), Citadini-Zanette; Baptista (1989), Diesel; Siqueira (1991), Andreata et al. (1997), Muller; Waechter (2001) e Kozera; Rodrigues (2005). Além desses, realizados em florestas brasileiras, Gentry; Dodson (1987), Poulsen e Balslev (1991), Poulsen; Pendry (1995), Poulsen (1996), Turner et al. (1996) e Laska (1997) também estudaram o estrato herbáceo-subarbustivo de áreas de floresta tropical, mas localizadas em outros países.

Tendo em vista a escassez de estudos no Brasil sobre o componente inferior de áreas de Floresta Ombrófila Densa (FOD), realizou-se o presente trabalho, que teve como objetivos listar as espécies de angiospermas e de pteridófitas e caracterizar a fisionomia do sub-bosque de uma área de Floresta Ombrófila Densa Montana. O estudo fez parte de um projeto maior que envolveu, além deste, o levantamento florístico e fitossociológico do estrato inferior de uma outra área de Floresta Ombrófila Densa (KOZERA; RODRIGUES, 2005).

\section{MATERIAL E MÉTODOS}

A área de estudo está localizada no Parque Estadual Pico do Marumbi (PEPM), porção central da vertente oriental do trecho paranaense da Serra do Mar (PARANÁ, 1996). Ele abriga uma das maiores áreas de Floresta Ombrófila Densa ainda preservadas do estado (Figura 1).

Dentro do PEPM, a área de estudo está situada num trecho da encosta do morro Facãozinho. É cortada por um riacho perene $\left(25^{\circ} 26^{\prime} \mathrm{S}\right.$ e $\left.48^{\circ} 55^{\prime} \mathrm{W}\right)$ e se encontra a cerca de $630 \mathrm{~m}$ s.n.m. (DITTRICH et al., 2005). Possui aproximadamente 2 ha e foi selecionada pelas condições de acesso e por concentrar outros estudos relacionados à vegetação, entre eles o levantamento florístico das espécies de angiospermas do componente epifítico (Cláudia Giongo, dados não publicados) e das pteridófitas terrícolas e epifíticas (DITTRICH et al., 2005).

Segundo o sistema de classificação climática de Köppen, o clima na área do PEPM enquadra-se no tipo Cfb (PARANÁ, 1996). Dados obtidos por Rocha (1999) durante 1997, coletados de uma estação meteorológica instalada na área da sede do Parque, a $485 \mathrm{~m}$ de altitude, registraram média anual de umidade relativa do ar de $90,7 \%$, sendo que nos meses de inverno as máximas diárias mantiveram-se entre 70 e $100 \%$ e nos demais meses em $100 \%$. A temperatura média anual foi de $19,7^{\circ} \mathrm{C}$, a temperatura 
média das máximas $25,2{ }^{\circ} \mathrm{C}$ e a mínima $16,2{ }^{\circ} \mathrm{C}$. Do total de precipitação registrada, $3.036 \mathrm{~mm}, 82 \%$ concentraram-se nos meses de primavera e verão e $18 \%$ nos meses de outono e inverno.
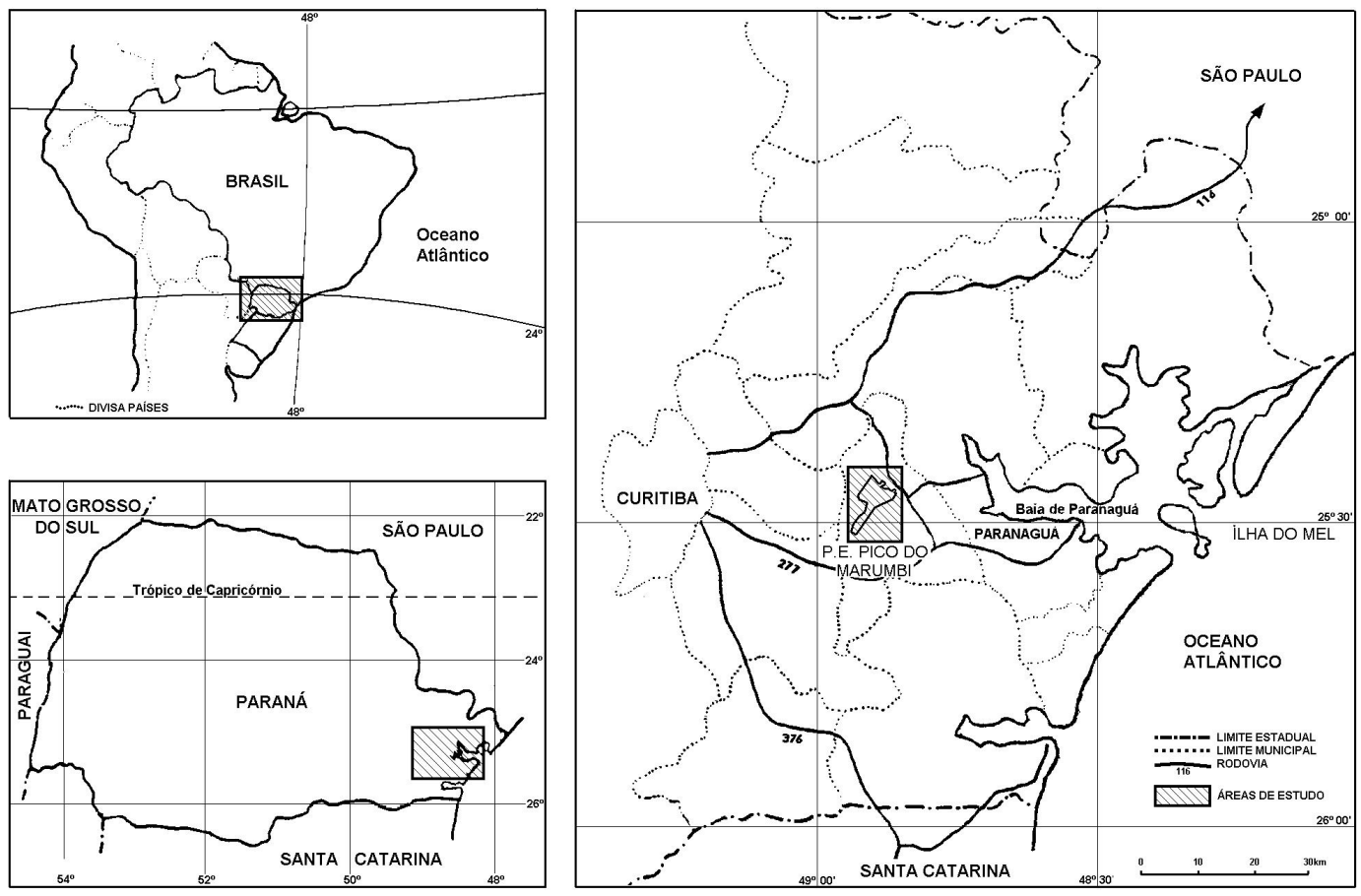

Figura 1. Localização da área de estudo, Parque Estadual Pico do Marumbi, Morretes (PR).

Figure 1. Location of the study area, Pico do Marumbi State Park, Morretes (PR).

Quanto aos tipos de solos, na área do estudo ocorrem Cambissolos, Neossolos e afloramentos de rocha, sendo esse último um tipo de terreno e não propriamente um solo, representado por exposições de rocha com reduzidas porções de materiais detríticos grosseiros (PARANÁ, 1996; EMBRAPA 1999).

Com relação à vegetação, a área é coberta por Floresta Ombrófila Densa Montana conservada (VELOSO et al., 1991; IBGE, 1992).

Para o estudo, foram coletadas todas as espécies de angiospermas e de pteridófitas presentes no sub-bosque da floresta. Foram incluídas no levantamento as herbáceas e as subarbustivas. As coletas foram realizadas quinzenalmente entre janeiro de 1999 e fevereiro de 2000. Para isso, percorria-se uma área previamente delimitada para o estudo, aleatoriamente ao longo de um dia e através de caminhadas, examinando-se os diferentes ambientes observados no local. A área percorrida iniciava nas margens direita e esquerda do riacho presente no local e seguia, aproximadamente, $100 \mathrm{~m}$ em direção às porções mais altas do trecho de encosta e ao longo de $100 \mathrm{~m}$ paralelamente ao curso do rio.

Os procedimentos adotados para a coleta e herborização seguiram as recomendações básicas para trabalhos dessa natureza (INSTITUTO DE BOTÂNICA, 1989; IBGE, 1992).

No momento da coleta foram anotadas informações referentes ao hábitat da espécie (terrícola: espécie que cresce enraizada no solo; rupícola: espécie que cresce sobre rochas ${ }^{1}$ ); hábito (herbácea: planta não lignificada; subarbustiva: planta arbustiva com até $1,5 \mathrm{~m}$ de altura e lignificada apenas na base); formas biológicas observadas em campo, segundo Silva (1998); coloração das peças florais e/ou frutos; e informações sobre o ambiente no qual foi encontrada.

Após a secagem, os materiais foram determinados em nível de família, gênero e espécie, através de bibliografia específica e comparações com exsicatas dos herbários UPCB (Departamento de Botânica, Setor de Ciências Biológicas, Universidade Federal do Paraná) e MBM (Museu Botânico Municipal de

$\overline{1}$ As rochas correspondem aos afloramentos, de tamanhos variados, dispostos aleatoriamente ao longo da área de estudo. 
Curitiba). Algumas duplicatas também foram enviadas a especialistas, para determinação e/ou confirmação da determinação.

Os materiais determinados foram registrados e incorporados na coleção do Herbário UEC (Universidade Estadual de Campinas). Duplicatas foram distribuídas para os Herbários ESA (Escola Superior de Agricultura "Luiz de Queiroz", Piracicaba, SP), UPCB e MBM.

Para a conferência da nomenclatura dos nomes científicos e dos respectivos autores, foram consultados os bancos de dados eletrônicos do Jardim Botânico de Missouri (MISSOURI BOTANICAL GARDEN, 2007) e do IPNI (IPNI - The International Plant Names Index, 2007).

O sistema de classificação adotado para as famílias de angiospermas foi o proposto por Cronquist (1988), e para as pteridófitas, subdivididas em samambaias e lycophytas, o sistema de Smith et al. (2006) e de Tryon; Tryon (1982), respectivamente.

\section{RESULTADOS}

Foram registradas 108 espécies em 68 gêneros e 41 famílias. Desse total, pertencem às angiospermas 70 espécies, 46 gêneros e 29 famílias (Tabela 1); às pteridófitas, 38 espécies, 22 gêneros e 12 famílias (Tabela 2). Cinco espécies são rupícolas, oito rupícolas e/ou terrícolas e 95 são terrícolas.

Tabela 1. Angiospermas do sub-bosque da Floresta Ombrófila Densa Montana do morro Facãozinho, Morretes, Paraná, com seus respectivos hábitats (TE: terrícola; RU: rupícola), hábitos (HE: herbácea; SUB: subarbustiva), formas biológicas observadas em campo (BU: bulbosa; CE: cespitosa; ER: ereta; RE: reptante; RI: rizomatosa; RO: rosulada; TR: trepadeira) e números de registro no herbário UEC.

Table 1. Angiosperms of the understory of a tropical rain forest at Facãozinho montain, Morretes, Paraná with habitat (TE: terrestrial; RU: lithophytes), life-form (HE: herbaceous; SUB: shrubby), growth form (BU: bulbous; CE: tufted; ER: erect; RE: creeping; RI: rhizomatous; RO: rosetted; TR: climber) and registry number at UEC.

\begin{tabular}{|c|c|c|c|c|c|}
\hline Família & Espécies & Hábitat & Hábito & $\begin{array}{l}\text { Forma } \\
\text { biológica }\end{array}$ & UEC \\
\hline \multirow[t]{5}{*}{ Acanthaceae } & Justicia carnea Lindl. & $\mathrm{TE}$ & $\mathrm{HE}$ & ER & 110048 \\
\hline & Justicia schenckiana Lindau & TE & $\mathrm{HE}$ & ER & 110049 \\
\hline & Pseuderanthemum riedelianum Nees & $\mathrm{TE}$ & $\mathrm{HE}$ & ER & 110050 \\
\hline & Ruellia solitaria Vell. & TE & $\mathrm{HE}$ & ER & 110051 \\
\hline & Staurogyne mandioccana Nees & $\mathrm{TE}$ & $\mathrm{HE}$ & ER & 110052 \\
\hline Amaranthaceae & Celosia grandifolia Moq. & TE & $\mathrm{HE}$ & ER & 110053 \\
\hline \multirow[t]{7}{*}{ Araceae } & Anthurium harrisii (Grah.) G. Don & TE, RU & $\mathrm{HE}$ & RO & 114847 \\
\hline & Anthurium pentaphyllum (Aubl.) G.Don & $\mathrm{TE}$ & $\mathrm{HE}$ & TR, RE & $* *$ \\
\hline & Monstera adansonii Schott & $\mathrm{TE}$ & $\mathrm{HE}$ & $\mathrm{TR}, \mathrm{RE}$ & $* *$ \\
\hline & Philodendron appendiculatum Nadruz e Mayo & TE & $\mathrm{HE}$ & TR, RE & 114849 \\
\hline & Philodendron loefgrenii Engl. & TE & $\mathrm{HE}$ & $\mathrm{TR}, \mathrm{RE}$ & 114848 \\
\hline & Philodendron ochrostemon Schott & TE & $\mathrm{HE}$ & TR, RE & 110055 \\
\hline & Philodendron sp. & TE & $\mathrm{HE}$ & TR, RE & $* *$ \\
\hline \multirow[t]{2}{*}{ Arecaceae } & Geonoma elegans Mart. & $\mathrm{TE}$ & SUB & RO & 110056 \\
\hline & Geonoma gamiova Barb. Rodr. & TE & SUB & RO & 110057 \\
\hline \multirow[t]{3}{*}{ Asteraceae } & Adenostemma brasilianum (Pers.) Cass. & $\mathrm{TE}$ & $\mathrm{HE}$ & ER & 110058 \\
\hline & Mikania cf. trinervis Hook. e Arn. & TE & $\mathrm{HE}$ & TR & $* *$ \\
\hline & Mikania sp. & $\mathrm{TE}$ & $\mathrm{HE}$ & $\mathrm{TR}$ & $* *$ \\
\hline \multirow[t]{5}{*}{ Begoniaceae } & Begonia echinosepala Regel & TE, RU & $\mathrm{HE}$ & ER & 110061 \\
\hline & Begonia itupavensis Brade & TE, RU & $\mathrm{HE}$ & ER & 110059 \\
\hline & Begonia paleata Schott ex A. DC. & $\mathrm{TE}$ & $\mathrm{HE}$ & ER & 110062 \\
\hline & Begonia radicans Vell. & $\mathrm{TE}$ & $\mathrm{HE}$ & TR & 110060 \\
\hline & Begonia sp. & TE & $\mathrm{HE}$ & ER & $* *$ \\
\hline \multirow[t]{3}{*}{ Bromeliaceae } & Nidularium campo-alegrensis Leme & TE & $\mathrm{HE}$ & RO & 110063 \\
\hline & Nidularium innocentii Lem. & TE & $\mathrm{HE}$ & RO & 110064 \\
\hline & Nidularium procerum Lindm. & TE & $\mathrm{HE}$ & RO & 110065 \\
\hline Campanulaceae & Siphocampylos convolvulaceus (Cham.) G. Don & $\mathrm{TE}$ & $\mathrm{HE}$ & ER & 110066 \\
\hline Commelinaceae & Dichorisandra hexandra (Aubl.) Standl. & TE & $\mathrm{HE}$ & RI & 114846 \\
\hline
\end{tabular}




\begin{tabular}{|c|c|c|c|c|c|}
\hline & Dichorisandra thyrsiflora J.C. Mikan & TE & HE & RI & 110070 \\
\hline & Dichorisandra sp. & TE & $\mathrm{HE}$ & RI & $* *$ \\
\hline Cyclanthaceae & Asplundia polymera (Hand.-Mazz.) Harling & TE, RU & $\mathrm{HE}$ & TR, RE & 110067 \\
\hline \multirow[t]{3}{*}{ Cyperaceae } & Pleurostachys gaudichaudii Brongn. & TE & $\mathrm{HE}$ & RI & 110068 \\
\hline & Pleurostachys urvillei Brongn. & $\mathrm{TE}$ & $\mathrm{HE}$ & RI & 110069 \\
\hline & Scleria panicoides Kunth & TE & HE & RI & 114858 \\
\hline Dioscoreaceae & Dioscorea sp. & $\mathrm{TE}$ & $\mathrm{HE}$ & $\mathrm{TR}$ & $* *$ \\
\hline Euphorbiaceae & Phyllanthus glaziovii Müll. Arg. & TE & SUB & ER & 110071 \\
\hline Gesneriaceae & $\begin{array}{l}\text { Napeanthus reitzii (L.B. Sm.) Burtt ex } \\
\text { Leeuwenb. }\end{array}$ & $\mathrm{TE}$ & $\mathrm{HE}$ & RO & 110072 \\
\hline Heliconiaceae & Heliconia velloziana L.A. Emygidio & $\mathrm{TE}$ & $\mathrm{HE}$ & RI & 110073 \\
\hline Iridaceae & Neomarica humilis (Klatt) Capellari Jr. & TE & HE & RI & $* *$ \\
\hline Liliaceae & Hyppeastrum aulicum Herb. & $\mathrm{TE}$ & $\mathrm{HE}$ & $\mathrm{BU}$ & 110054 \\
\hline Marantaceae & Calathea communis Wanderley e S. Vieira & TE & HE & RI & $1372 *$ \\
\hline \multirow[t]{2}{*}{ Melastomataceae } & Bertolonia acuminata Gardner & TE & $\mathrm{HE}$ & ER & 110074 \\
\hline & Bertolonia mosenii Cogn. & $\mathrm{TE}$ & $\mathrm{HE}$ & ER & 110075 \\
\hline Myrsinaceae & Ardisia guianensis (Aubl.) Mez. & TE & SUB & ER & 110077 \\
\hline Moraceae & Dorstenia carautae C.C. Berg & $\mathrm{TE}$ & $\mathrm{HE}$ & ER & 110076 \\
\hline \multirow[t]{2}{*}{ Orchidaceae } & Pelexia hypnophila (Barb. Rodr.) Schltr. & TE & HE & RO & 114857 \\
\hline & Promenaea stapelioides (Link e Otto) Lindl. & RU & $\mathrm{HE}$ & $\mathrm{RE}$ & 114859 \\
\hline \multirow[t]{6}{*}{ Piperaceae } & Ottonia martiana Miq. & TE & SUB & ER & 110078 \\
\hline & Piper caldense C.DC. & $\mathrm{TE}$ & SUB & ER & 110079 \\
\hline & Piper cernuum Vell. & TE & SUB & ER & 110080 \\
\hline & Piper dilatatum Rich. & TE & SUB & ER & 110082 \\
\hline & Piper gaudichaudianum Kunth & TE & SUB & ER & 110081 \\
\hline & Piper $\mathrm{cf}$. lucaeanum var. grandifolium Yunck. & TE & SUB & ER & 114852 \\
\hline \multirow[t]{4}{*}{ Poaceae } & Olyra glaberrima Raddi & $\mathrm{TE}$ & $\mathrm{HE}$ & $\mathrm{CE}$ & 110083 \\
\hline & $\begin{array}{l}\text { Parodiolyra micrantha (Kunth) Davidse e } \\
\text { Zuloaga }\end{array}$ & $\mathrm{TE}$ & $\mathrm{HE}$ & $\mathrm{CE}$ & 110084 \\
\hline & Panicum ovuliferum Trin. & $\mathrm{TE}$ & $\mathrm{HE}$ & $\mathrm{RE}$ & 114850 \\
\hline & Panicum pilosum $\mathrm{Sw}$. & $\mathrm{TE}$ & $\mathrm{HE}$ & $\mathrm{CE}$ & 114851 \\
\hline \multirow[t]{6}{*}{ Rubiaceae } & Chomelia brasiliana A. Rich. & TE & SUB & ER & 110085 \\
\hline & Faramea hyacinthina Mart. & $\mathrm{TE}$ & SUB & ER & 110086 \\
\hline & Hoffmannia peckii K. Schum. & TE & $\mathrm{HE}$ & ER & 110087 \\
\hline & Psychotria birotula L.B. Sm. e Downs & $\mathrm{TE}$ & SUB & ER & 110088 \\
\hline & Psychotria brachypoda (Müll. Arg.) Britton & TE & SUB & ER & 110089 \\
\hline & Psychotria leiocarpa Cham. e Schltr. & TE & SUB & ER & 110090 \\
\hline Smilacaceae & Smilax sp. & TE & $\mathrm{HE}$ & TR & $* *$ \\
\hline \multirow[t]{4}{*}{ Solanaceae } & Brunfelsia pauciflora (Cham. E Schltdl.) Benth. & $\mathrm{TE}$ & SUB & ER & 110091 \\
\hline & Capsicum lucidum (Moric.) Kuntze & TE & SUB & ER & 110092 \\
\hline & Cestrum amictum Schltdl. & $\mathrm{TE}$ & SUB & ER & 110093 \\
\hline & Solanum rivulare Mart. & TE & SUB & ER & 110094 \\
\hline Urticaceae & Pilea artrogramma Miq. & TE, RU & $\mathrm{HE}$ & ER & 110095 \\
\hline Violaceae & Noisettia orchidiflora (Rudge) Gingins & $\mathrm{TE}$ & $\mathrm{HE}$ & ER & 110096 \\
\hline
\end{tabular}

Dentre as angiospermas, destacaram-se por apresentar os maiores números de espécies, Araceae (7), Piperaceae (6), Rubiaceae (6), Acanthaceae (5), Begoniaceae (5), Poaceae (4) e Solanaceae (4). Essas famílias correspondem a 53\% do total de espécies de angiospermas amostradas. Dentre as pteridófitas, Dryopteridaceae (11), Blechnaceae (6), Cyatheaceae (4) e Hymenophyllaceae (4) foram as mais ricas, perfazendo $65 \%$ do total de espécies registradas para esse grupo de plantas. Quanto ao hábito, são dominantes as herbáceas (85), seguidas pelas subarbustivas (23). Nesse último grupo foram ainda incluídas as palmeiras (Arecaceae) de pequeno porte e as pteridófitas arborescentes.

As angiospermas foram representadas por sete formas biológicas e as pteridófitas por quatro. No cômputo geral, predominaram espécies eretas, rosuladas e rizomatosas (Figura 2).

Ao longo da área de estudo foram observados, com frequência, afloramentos de rocha de tamanhos variados, dispostos erraticamente, e depósitos de material aluvionar, representados por grande quantidade de seixos, principalmente nas margens e no leito do riacho. Sobre esses fragmentos de rocha e 
sobre os clastos ocorrem diferentes espécies de plantas herbáceas, entre elas Pilea artrogramma Miq. e Calathea communis Wanderley e S. Vieira, a primeira com crescimento ereto e a segunda formando grandes touceiras rizomatosas. Em meio a elas, ainda podem ser observadas outras espécies, como Begonia paleata Schott ex A. DC., Celosia grandifolia Moq., Dichorisandra thyrsiflora J.C. Mikan, Danaea moritziana C. Presl, Diplazium ambiguum Raddi, Hoffmannia peckii K. Schum., Justicia carnea Lindl., Megalastrum connexum (Kaulf.) A.R. Sm. e R.C. Moran, Noisetia orchidiflora (Rudge) Gingins, Asplenium triquetrum N. Murak. e R.C. Moran e Dennstaedtia dissecta (Sw.) T. Moore.

Tabela 2. Pteridófitas do sub-bosque da Floresta Ombrófila Densa Montana do morro Facãozinho, Morretes, Paraná, com seus respectivos hábitat (TE: terrícola, RU: rupícola), hábito (HE: herbácea, SUB: subarbustiva), forma biológica observada em campo (RE: reptante, RI: rizomatosa, RO: rosulada, TR: trepadeira) e número de registro no herbário UEC.

Table 2. Pteridophytes of the understory of a tropical rain forest at Facãozinho, mountain, Morretes, Paraná, with habitat (TE: terrestrial, RU: Lithophytes), life-form (HE: herbaceous, SUB: subshrub), growth form (RE: creeping, RI: rhizomatous, RO: rosetted, TR: climber) and registry number at UEC.

\begin{tabular}{|c|c|c|c|c|c|}
\hline Família & Espécie & Hábitat & Hábito & $\begin{array}{l}\text { Forma } \\
\text { biológica }\end{array}$ & UEC \\
\hline \multirow[t]{2}{*}{ Aspleniaceae } & Asplenium kunzeanum Klotzsch ex Rosenst. & TE, RU & $\mathrm{HE}$ & RO & 110098 \\
\hline & Asplenium triquetrum N. Murak.e R.C. Moran & RU & HE & RI & 110097 \\
\hline \multirow[t]{6}{*}{ Blechnaceae } & $\begin{array}{l}\text { Blechnum binervatum subsp. Acutum (Desv.) R.M. } \\
\text { Tryon e Stolze }\end{array}$ & RU & $\mathrm{HE}$ & RI & $* *$ \\
\hline & Blechnum brasiliense Desv. & $\mathrm{TE}$ & HE & RO & 110101 \\
\hline & Blechnum divergens (Kunze) Mett. & TE & $\mathrm{HE}$ & RO & 110100 \\
\hline & Blechnum lehmannii Hieron. & $\mathrm{TE}$ & HE & RO & 114855 \\
\hline & Blechnum sampaioanum Brade & $\mathrm{TE}$ & HE & RO & 110099 \\
\hline & Salpichlaena volubilis (Kaulf.) J. Sm. & $\mathrm{TE}$ & HE & TR & 114856 \\
\hline \multirow[t]{4}{*}{ Cyatheaceae } & $\begin{array}{l}\text { Alsophila sternbergii (Pohl ex Sternb.) D.S. } \\
\text { Conant }\end{array}$ & TE & SUB & RO & $* *$ \\
\hline & Cyathea corcovadensis (Raddi) Domin & TE & SUB & RO & 110102 \\
\hline & Cyathea leucofolis Domin & $\mathrm{TE}$ & SUB & RO & 110103 \\
\hline & Cyathea phalerata Mart. & $\mathrm{TE}$ & SUB & RO & 110105 \\
\hline Dennstaedtiaceae & Dennstaedtia dissecta (Sw.) T. Moore & RU & HE & RI & 110106 \\
\hline \multirow{11}{*}{ Dryopteridaceae } & Ctenitis anniesii (Rosenst.) Copel. & TE & HE & RO & $39911^{*}$ \\
\hline & Ctenitis deflexa (Kaulf.) Copel. & TE & HE & RO & 110107 \\
\hline & Ctenitis pedicellata (H. Christ) Copel. & $\mathrm{TE}$ & $\mathrm{HE}$ & RO & 110108 \\
\hline & Lastreopsis amplissima (C. Presl) Tindale & $\mathrm{TE}$ & HE & RI & 110112 \\
\hline & Lomagramma guianensis (Aubl.) Ching & TE & HE & RI & 110113 \\
\hline & $\begin{array}{l}\text { Megalastrum connexum (Kaulf.)A.R. Sm. e R.C. } \\
\text { Moran }\end{array}$ & $\mathrm{TE}$ & $\mathrm{HE}$ & RO & 110114 \\
\hline & Olfersia cervina (L.) Kunze & TE, RU & HE & RI & 110115 \\
\hline & Polybotrya cylindrica Kaulf. & TE & HE & RI & 110104 \\
\hline & Stigmatopteris brevinervis (Fée) R. C. Moran & TE & HE & RO & 110116 \\
\hline & Stigmatopteris caudata (Raddi) C. Chr. & $\mathrm{TE}$ & HE & RO & 110117 \\
\hline & Stigmatopteris heterocarpa (Fée) Rosenst. & $\mathrm{TE}$ & HE & RO & 110118 \\
\hline \multirow[t]{4}{*}{ Hymenophyllaceae } & Hymenophyllum caudiculatum Mart. & RU & HE & RI & 114854 \\
\hline & Trichomanes collariatum Bosch & TE & HE & RI & 110120 \\
\hline & Trichomanes pyxidiferum $\mathrm{L}$. & TE & HE & RI & $* *$ \\
\hline & Trichomanes rigidum $\mathrm{Sw}$. & TE & $\mathrm{HE}$ & RI & 110121 \\
\hline \multirow[t]{3}{*}{ Marattiaceae } & Danaea elliptica $\mathrm{Sm}$. & TE & $\mathrm{HE}$ & RO & 110122 \\
\hline & Danaea moritziana C. Presl & TE & HE & RO & 110123 \\
\hline & Marattia cicutifolia Kaulf. & TE & HE & RO & 110124 \\
\hline Polypodiaceae & Campyloneurum minus Fee & TE, RU & HE & RI & 110126 \\
\hline Pteridaceae & Pteris decurrens C. Presl & TE & $\mathrm{HE}$ & RO & 110125 \\
\hline Selaginellaceae & Selaginella flexuosa Spring & TE & $\mathrm{HE}$ & $\mathrm{RE}$ & 114853 \\
\hline Tectariaceae & Tectaria incisa Cav. & TE & $\mathrm{HE}$ & $\mathrm{RI}$ & 110119 \\
\hline \multirow[t]{3}{*}{ Woodsiaceae } & Diplazium ambiguum Raddi & TE & HE & RO & 110109 \\
\hline & Diplazium cristatum (Desr.) Alston & TE & $\mathrm{HE}$ & RO & 110110 \\
\hline & Diplazium plantaginifolium (L.) Urb. & TE & $\mathrm{HE}$ & RI & 110111 \\
\hline
\end{tabular}

*: número de registro no herbário UPCB (Curitiba - PR); **: sem coleta de material fértil e/ou sem número de registro do herbário UEC. 


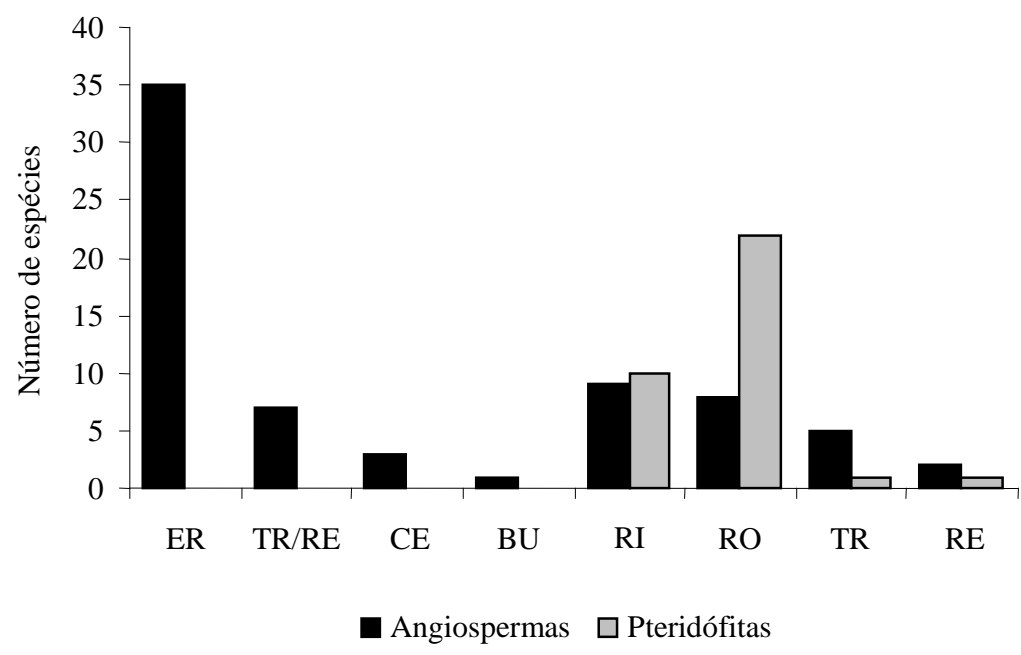

Figura 2. Número de espécies de angiospermas e de pteridófitas do sub-bosque de uma Floresta Ombrófila Densa Montana, morro Facãozinho, Morretes (PR). (Formas biológicas: ER: ereta; TR: trepadeira; RE: reptante; CE: cespitosa; BU: bulbosa; RI: rizomatosa; RO: rosulada).

Figure 2. Angiosperms and pterydophyte species number of a tropical rain forest at Facãozinho montain, Morretes (PR). (Growth forms: ER: erect; TR: climber; RE: creeping; CE: tufted; BU: bulbous; RI: rhizomatous; RO: rosetted).

Nessa área também é bastante comum a ocorrência de Philodendron ochrostemon Schott (Araceae), uma hemiepífita secundária observada neste estudo como planta trepadeira ou como herbácea com crescimento reptante sobre o solo, condição relacionada à fase de seu desenvolvimento que antecede àquela que ocorre sobre os forófitos e sem contato nutricional com o solo. Essa espécie se destaca na fisionomia do sub-bosque por ocorrer em abundância e recobrindo grande parte da área estudada. Foram observadas grandes populações desse táxon sobre solos hidromórficos e grupos menores e mais esparsos nas partes mais altas da encosta, em ambientes mais bem drenados. Asplundia polymera (Hand.-Mazz.) Harling, uma Cyclanthaceae, também ocorre próxima do riacho, sendo, no entanto, mais comum como trepadeira em árvores de grande porte.

Nas áreas mais íngremes, ainda localizadas ao longo do riacho, foram observadas algumas espécies que parecem seletivas desses ambientes, não tendo sido encontradas em outros locais na mesma área. Entre elas estão Napeanthus reitziii (L.B. Sm.) Burtt ex Leeuwenb., uma Gesneriaceae herbácea de pequeno porte e com flores azuladas, e as pteridófitas Blechnum lehmannii Hieron. e Selaginella flexuosa Spring. Além da limitação imposta pela declividade do terreno, com consequente adelgamento do solo, essas espécies também se encontram em condições de restrição quanto à luminosidade.

No início da encosta, próximos ao riacho, ocorrem agrupamentos de pteridófitas arborescentes, muitas das quais com altura superior a $5 \mathrm{~m}$. Entre elas, são relativamente comuns Alsophila sternbergii (Pohl ex Sternb.) D.S. Conant e Cyathea phalerata Mart. Já nas porções mais afastadas, onde o solo é mais bem drenado e a declividade mais acentuada, predominam pteridófitas com crescimento rosulado ou rizomatoso, tais como Stigmatopteris heterocarpa (Fée) Rosenst., Lastreopsis amplissima (C. Presl) Tindale, Pteris decurrens C. Presl, Blechnum sampaioanum Brade, Danaea moritziana C. Presl, Polybotrya cylindrica Kaulf. e Lomagramma guianensis (Aubl.) Ching. As características hídricas e edáficas do ambiente provavelmente estão entre as determinantes das suas respectivas distribuições que, em parte, contribuem para o aspecto de heterogeneidade da fisionomia do sub-bosque.

Ainda com relação à distribuição das espécies, algumas foram observadas de forma bastante irregular na área de estudo, ocorrendo isoladamente ou reunidas em pequenos agrupamentos ao longo da encosta. Entre essas estão Anthurium harrisii (Grah.) G. Don, Begonia itupavensis Brade, Ctenitis deflexa (Kaulf.) Copel., Ctenitis pedicellata (H. Christ) Copel., Diplazium plantaginifolium (L.) Urb., Dorstenia 
carautae C.C. Berg., Marattia cicutifolia Kaulf., Nidularium campoalegrense Leme, Nidularium innocentii Lem., Olyra glaberrima Raddi, Pleurostachys gaudichaudii Brongn., Pleurostachys urvillei Brongn., Psychotria birotula L.B. Sm. e Downs, Psychotria brachypoda (Müll. Arg.) Britton, Ruellia solitaria Vell. e Staurogyne mandioccana Nees.

Em meio às herbáceas, também foram observadas algumas espécies subarbustivas, destacando-se dentre elas Ardisia guianensis (Aubl.) Mez, Brunfelsia pauciflora (Cham. e Schltdl.) Benth., Faramea hyacinthina Mart., Geonoma elegans Mart., Geonoma gamiova Barb. Rodr. e Piper cf. lucaeanum var. grandifolium Yunck. Essas espécies subarbustivas se encontram presentes tanto no estrato inferior (subbosque) como naquele imediatamente superior, representado pelas espécies arbustivas.

Dentro da área de estudo, também foram observadas espécies herbáceas e subarbustivas em duas pequenas clareiras formadas pela queda de árvores de grande porte. Nesses locais, em especial, entre outras espécies da regeneração natural, foram encontradas Blechnum brasiliense Desv., Capsicum lucidum (Moric.) Kuntze, Parodiolyra micrantha (Kunth) Davidse e Zuloaga, Panicum pilosum Sw., Panicum ovuliferum Trin., Salpichlaena volubilis (Kaulf.) J. Sm. e Scleria panicoides Kunth, espécies não observadas em outros ambientes no interior da floresta e com crescimento favorecido principalmente pelas condições de maior luminosidade, originadas pela abertura do dossel.

\section{DISCUSSÃO}

O elevado número de espécies herbáceas e subarbustivas registradas no levantamento florístico demonstra o quão rico é o sub-bosque estudado. A contribuição efetiva das espécies para a riqueza da área é alta, tanto em termos numéricos quanto em formas de vida.

Silva (1989), utilizando metodologia de pontos para amostragem do componente arbóreo de uma área de FOD bem conservada, também localizada no PEPM e a cerca de 500 m s.n.m., encontrou, após a instalação de pontos (método fitossociológico) com distância de $10 \mathrm{~m}$ entre eles, 70 espécies de 31 famílias. Melo; Mantovani (1994), em uma área de FOD de encosta no Parque Estadual da Ilha do Cardoso, encontraram 157 espécies e 47 famílias entre árvores com perímetro à altura do peito (PAP) maior ou igual a $8 \mathrm{~cm}$, palmeiras e fetos arborescentes com caule maior do que $2 \mathrm{~m}$ de altura. GuedesBruni et al. (1997), em FOD Montana em bom estado de conservação, localizada na Reserva Ecológica de Macaé de Cima (RJ), encontraram 189 espécies e 48 famílias, entre árvores com PAP maior ou igual a $5 \mathrm{~cm}$, palmeiras e fetos arborescentes. Os resultados desses trabalhos (Tabela 3), relacionados aos estratos superiores de FOD, estão muito próximos dos que foram obtidos neste estudo, evidenciando, com isso, que as espécies que compõem o estrato inferior da floresta contribuem para a riqueza das áreas de FOD tanto quanto as arbóreas. Com isso, ressalta-se a importância florística dessa sinúsia na composição da respectiva formação vegetal.

Andreata et al. (1997), da mesma forma como observado neste estudo, também encontraram elevado número de espécies no sub-bosque de uma FOD na Reserva Ecológica de Macaé de Cima (RJ). Esses autores, em levantamento de parcelas mais algumas coletas aleatórias, efetuadas dentro dos limites da Reserva, registraram 249 táxons pertencentes a 43 famílias, com destaque para Orchidaceae (46), Rubiaceae (37), Melastomataceae (33) e Piperaceae (17). No trabalho não foram incluídas as pteridófitas e dentre as arbustivas foram consideradas todas as angiospermas com até $4 \mathrm{~m}$ de altura (Tabela 3 ).

Tabela 3. Estudos em áreas de Floresta Ombrófila Densa.

Table 3. Studies in areas of Dense Ombrophilous Montane Forest.

\begin{tabular}{|c|c|c|c|c|c|c|}
\hline Referência & Local & Amostragem & Vegetação & Estrato & Espécies & Famílias \\
\hline Silva (1989) & PEPM, Morretes, PR & $\begin{array}{l}80 \text { pontos, } \\
\text { DAP }>=4,7 \mathrm{~cm}\end{array}$ & FOD de encosta & arbóreo & 70 & 31 \\
\hline $\begin{array}{l}\text { Melo e Mantovani } \\
\text { (1994) }\end{array}$ & PEIC, Cananeia, SP & $\begin{array}{l}1 \text { parcela } 1 \mathrm{ha}, \\
\text { PAP }>=8 \mathrm{~cm}\end{array}$ & FOD de encosta & arbóreo & 157 & 47 \\
\hline $\begin{array}{l}\text { Guedes-Bruni et } \\
\text { al. (1997) }\end{array}$ & $\begin{array}{l}\text { REMC, } \\
\text { Nova Friburgo, RJ }\end{array}$ & $\begin{array}{l}1 \text { parcela } 1 \mathrm{ha}, \\
\text { PAP }>=5 \mathrm{~cm}\end{array}$ & FOD Montana & arbóreo & 189 & 48 \\
\hline $\begin{array}{l}\text { Andreata et al. } \\
\text { (1997) }\end{array}$ & $\begin{array}{l}\text { REMC, } \\
\text { Nova Friburgo, RJ }\end{array}$ & 2 parcelas 1 ha & FOD Montana & $\begin{array}{l}\text { herbáceo e } \\
\text { arbustivo }\end{array}$ & 249 & 43 \\
\hline
\end{tabular}

PEPM: Parque Estadual Pico do Marumbi; PEIC: Parque Estadual Ilha do Cardoso; REMC: Reserva Ecológica de Macaé de Cima; FOD: Floresta Ombrófila Densa; DAP: diâmetro à altura do peito; PAP: perímetro à altura do peito. 
Além da contribuição em termos de riqueza para a floresta, as espécies herbáceas e subarbustivas também são importantes no ambiente em que ocorrem, atuando como indicadoras da qualidade do meio (RICHARDS, 1952). O pequeno porte e a concorrência com as espécies arbóreas, principalmente com relação aos fatores ambientais, tornam-nas sensíveis às diferenças do ambiente, condições nas quais os vegetais de maior porte não manifestam reação (CITADINI-ZANETTE, 1984).

No morro Facãozinho, área deste estudo, a diversidade de ambientes presentes, ocupados por espécies adaptadas às características desses locais, pode ser citada como um exemplo da estreita relação das espécies herbáceas e subarbustivas com o meio no qual estão estabelecidas. Sobre as rochas nas margens do riacho, nas superfícies aluvionares e nas encostas mais ou menos íngremes, expostas a valores variáveis de incidência luminosa, ocorrem diferentes conjuntos de espécies, conforme descrito anteriormente.

A riqueza de ambientes, em conjunto com as espécies características do interior florestal, também contribui para a determinação da fisionomia do sub-bosque, que parece ao observador, num primeiro instante, bastante heterogênea.

A relação entre as espécies arbóreas e as herbáceas e/ou subarbustivas, em especial, ocorre através de variadas adaptações, principalmente relacionadas com a intensidade solar e as condições microclimáticas que se estabelecem no ambiente pelas árvores (MANTOVANI, 1987). Essas condições, em parte, são determinadas pela forma como o dossel florestal se apresenta entre o sub-bosque e o meio exterior, acima das copas, definido subjetivamente como dossel mais "fechado" ou "aberto" (GRUBB et al., 1963). Com isso, a estratificação florestal e a densidade das copas acabam determinando diferentes comportamentos do dossel na função de filtragem da luz para o sub-bosque, indicando a existência de um gradiente luminoso em virtude das inúmeras barreiras transpostas pelos raios luminosos, que aumentam a difusão e a reflexão (ZICKEL, 1995).

Nesse contexto, as diferentes formas biológicas registradas neste trabalho podem ser citadas como exemplos de como as espécies buscam otimizar o espaço do estrato inferior, ocupando os diferentes ambientes e procurando, assim, se adaptar às condições físicas do meio. As plantas trepadeiras, as reptantes e as rizomatosas, por exemplo, podem explorar o meio em que se encontram deslocando o sentido do seu crescimento em direção às áreas mais iluminadas, enquanto que as rosuladas e as cespitosas podem captar maior intensidade luminosa através de suas folhas que, em geral, são longas, largas e dispostas de forma espiralada.

Processos estocásticos e a heterogeneidade espaço-temporal, associados aos fatores bióticos, como competição, predação e parasitismo, e aos abióticos, como tipos de solo e a disponibilidade hídrica, também contribuem para a riqueza do estrato inferior de florestas tropicais (DIRZO et al., 1992). Por isso, é de fundamental importância a realização de levantamentos florísticos mais detalhados. Estudos dessa natureza, que futuramente venham a ser realizados, poderão fornecer, em conjunto, dados e informações mais precisas sobre a real diversidade das áreas de florestas tropicais brasileiras.

\section{REFERÊNCIAS}

ANDRADE, P. M. de. Estrutura do estrato herbáceo de trechos da Reserva Biológica Mata do Jambreiro, Nova Lima, MG. Dissertação (Mestrado) - Universidade Estadual de Campinas, Campinas, 1992.

ANDREATA, R. H. P.; GOMES, M.; BAUMGRATZ, J. F. A. Plantas herbáceo-arbustivas da Reserva Ecológica de Macaé de Cima. In: LIMA, H. C. de; GUEDES-BRUNI, R. R. (Eds.). Serra de Macaé de Cima: diversidade florística e conservação em Mata Atlântica. Rio de Janeiro: Jardim Botânico do Rio de Janeiro, 1997. p. 139-152.

BERNACCI, L. C. Estudo florístico e fitossociológico de uma floresta no município de Campinas, com ênfase nos componentes herbáceo e arbustivo. Dissertação (Mestrado) - Universidade Estadual de Campinas, Campinas, 1992.

CERVI, A. C.; ACRA, L. A.; RODRIGUES, J. L.; TRAIN, S.; IVANCHECHEN, S. L.; MOREIRA, A. L. O. R. Contribuição ao conhecimento das pteridófitas de uma mata de Araucária, Curitiba, Paraná, Brasil. Acta Biológica Paranaense, Curitiba, v. 16, n. 1-4, p. 77-85, 1987. 
CERVI, A. C.; ACRA, L. A.; RODRIGUES, L.; GABRIEL, M. M.; LOPES, M. Contribuição ao conhecimento das plantas herbáceas de uma floresta de Araucária do primeiro planalto paranaense. Ínsula, Florianópolis, v. 18, p. 83-98, 1988.

CESTARO, L. A.; WAECHTER, J. L.; BAPTISTA, L. R. de M. Fitossociologia do estrato herbáceo da mata de Araucária da Estação Ecológica de Aracuri, Esmeralda, RS. Hoehnea, São Paulo, v. 13, p. 59-72, 1986.

CITADINI-ZANETTE, V. Composição florística e fitossociológica da vegetação herbácea terrícola de uma mata de Torres, Rio Grande do Sul, Brasil. Iheringia, Porto Alegre, v. 32, p. 23-62, 1984.

CITADINI-ZANETTE, V.; BAPTISTA, L. R. de M. Vegetação herbácea terrícola de uma comunidade florestal em Limoeiro, município de Torres, Rio Grande do Sul, Brasil. Boletim do Instituto de Biociências, Porto Alegre, v. 45, p. 1-87, 1989.

CRONQUIST, A. The evolution and classification of flowering plants. New York: New York Botanical Garden, 1988.

DIESEL, S.; SIQUEIRA, J. C. de. Estudo fitossociológico herbáceo/arbustivo da mata ripária da bacia hidrográfica do Rio dos Sinos, Rio Grande do Sul. Pesquisas. Botânica, São Leopoldo, v. 42, p. 205-257, 1991.

DIRZO, R.; HORVITZ, C. C.; QUEVEDO, H.; LÓPEZ, M. A. The effects of gap size and age on the understorey herb community of a tropical Mexican rain forest. Journal of Ecology, Oxford, v. 80, p. 809$822,1992$.

DITTRICH, V. A. O.; WAECHTER, J. L.; SALINO, A. Species richness of pteridophytes in a montane Atlantic rain forest plot of Southern Brazil. Acta Botanica Brasilica, Porto Alegre, v. 19, n. 3, p. 519$525,2005$.

EMBRAPA. Sistema Brasileiro de classificação de solos. Brasília, DF: Centro Nacional de Pesquisa de Solos, 1999.

FIDALGO, O.; BONONI, V. L. R. (Coords.). Técnicas de coleta, preservação e herborização de material botânico. São Paulo: Instituto de Botânica, 1989. (Série Documentos).

GENTRY, A. H. Changes in plant community diversity and floristic composition on environmental and geographical gradients. Annals of the Missouri Botanical Garden, Saint Louis, v. 75, n. 1, p. 1-34, 1988.

GENTRY, A. H.; DODSON, C. Contribution of nontrees to species richness of a tropical rain forest. Biotropica, Washingtom, DC, v. 19, n. 2, p. 149-156, 1987.

GENTRY, A. H.; EMMONS, L. H. Geographical variation in fertility, phenology, and composition of the understory of neotropical forests. Biotropica, Washingtom, DC, v. 19, n. 3, p. 216-227, 1987.

GRUBB, P. J.; LLOYD, J. R.; PENNINGTON, T. D.; WHITMORE, T. C. A comparison of montane and lowland rain forest in Ecuador. Journal of Ecology, Oxford, v. 51, n. 3, p. 567-601, 1963.

GUEDES-BRUNIN, R. R.; PESSOA, S. de V. A.; KURTZ, B. C. Florística e estrutura do componente arbustivo-arbóreo de um trecho preservado de floresta montana na Reserva Ecológica de Macaé de Cima. In: LIMA, H. C. de; GUEDES-BRUNI, R. R. (Eds.). Serra de Macaé de Cima: diversidade florística e conservação em Mata Atlântica. Rio de Janeiro: Jardim Botânico do Rio de Janeiro, 1997. p. 127-146.

INSTITUTO BRASILEIRO DE GEOGRAFIA E ESTATÍSTICA (IBGE). Manual técnico da vegetação brasileira. Rio de Janeiro: IBGE, 1992.

IPNI - THE INTERNATIONAL PLANT NAMES INDEX. The international plant names index database. Disponível em: http://www.ipni.org/index.html. Acesso em janeiro 2007. 
KOZERA, C.; RODRIGUES, R. R. Floresta Ombrófila Densa Submontana: florística e estrutura do estrato inferior. In: Marques, M. C. M.; Britez, R. M. (Org.). História natural e conservação da Ilha do Mel. Curitiba: Editora da Universidade Federal do Paraná, 2005. p. 103-122.

LASKA, M. S. Structure of understory shrub assemblages in adjacent secondary and old growth tropical wet forest, Costa Rica. Biotropica, Washingtom, DC, v. 29, n. 1, p. 29-37, 1997.

MANTOVANI, W. Análise florística e fitossociológica do estrato herbáceo - subarbustivo do cerrado na Reserva Biológica de Moji Guaçu e em Itirapina. Tese (Doutorado) - Universidade Estadual de Campinas, Campinas, 1987.

MANTOVANI, W. Dinâmica da Floresta Pluvial Atlântica. In: WATANABE, S. (Coord.). SIMPÓSIO DE ECOSSISTEMAS BRASILEIROS, 4., 1998, São Paulo. Anais do IV. São Paulo: ACIESP, 1998. v. 2, p. 1-20.

MELO, M. M. F. de; MANTOVANI, W. Composição florística e estrutura de trecho de Mata Atlântica de encosta na Ilha do Cardoso, Cananéia, SP, Brasil. Boletim do Instituto de Botânica, São Paulo, v. 9, p. 107-158, 1994.

MISSOURI BOTANICAL GARDEN. Missouri Botanical Garden's VAST (Vascular Tropicos) nomenclatural database and associated authority files. Disponível em: <http://mobot.mobot.org/W3T/Search/vast.html>. Acesso em: 15/01/2007.

MORI, S. A. Eastern, Extra-Amazonian Brazil. In: CAMPBELL, D. G.; HAMMOND, H. D. (Eds.). Floristic inventory of tropical countries. New York: New York Botanical Garden, 1989. p. 427-454.

MÜLLER, S. C.; WAECHTER, J. L. Estrutura sinusial dos componentes herbáceo e arbustivo de uma floresta costeira subtropical. Revista Brasileira de Botânica, São Paulo, v. 24, n. 4, p. 395-406, 2001.

OGDEN, J.; POWELL, J. A. A quantitative description of the forest vegetation on an altitudinal gradient in the Mount Field National Park, Tasmania, and a dicussion on its history and dynamics. Australian Journal of Ecology, [S.1.], v. 4, p. 293-325, 1979.

PARANÁ. Plano de manejo do Parque Estadual Pico do Marumbi. Curitiba: Instituto Ambiental do Paraná, 1996.

POULSEN, A. D.; BALSLEV, H. Abundance and cover of ground herbs in an Amazonian rain forest. Journal of Vegetation Science, Knivsta, v. 2, p. 315-322, 1991.

POULSEN, A. D.; PENDRY, C. A. Inventories of ground herbs at three altitudes on Bukit Belalong, Brunei, Borneo. Biodiversity and Conservation, London, v. 4, p. 745-757, 1995.

POULSEN, A. D. Species richness and density of ground herbs within a plot of lowland rainforest in north-west Borneo. Journal of Tropical Ecology, Cambridge, v. 12, p. 177-190, 1996.

PRANCE, G. T. American tropical forest. In: LIETH, H.; WERGER, M. A. J. (Ed.). Tropical rain forest ecosystems: biogeographical and ecological studies. Amsterdam: Elsevier, 1989. p. 99-136.

PRANCE, G. T.; CAMPBELL, D. G. The present state of tropical floristics. Taxon, Utrecht, v. 37, n. 3, p. 519-548, 1988.

REEDER, W.; RIECHERT, S. E. Vegetation change along an altitudinal gradient, Santa Cruz Island, Galapagos. Biotropica, Washingtom, DC, v. 7, n. 3, p. 162-175, 1975.

RICHARDS, P. W. The tropical rain forest. Cambridge: Cambridge University Press, 1952.

ROCHA, M. do R. L. Caracterização fitossociológica e pedológica de uma Floresta Ombrófila Densa Altomontana no Parque Estadual Pico do Marumbi, Morretes, PR. 81 f. Dissertação (Mestrado em Engenhara Florestal) - Setor de Ciências Agrárias, Universidade Federal do Paraná, Curitiba, 1999. 
SILVA, F. C. da. Composição florística e estrutura fitossociológica da floresta tropical ombrófila da encosta atlântica no município de Morretes (Paraná). Boletim de Pesquisa Florestal, Colombo, n. 18/19, p. 31-49, 1989.

SILVA, S. M. As formações vegetais da planície litorânea da Ilha do Mel, Paraná: composição florística e principais características estruturais. Tese (Doutorado) - Universidade Estadual de Campinas, Campinas, 1998.

SMITH, A. P. Respuestas de hierbas del sotobosque tropical a claros ocasionados por la caída de árboles. Revista de Biologia Tropical, San Jose, v. 35 (supl. 1), p. 111-118, 1987.

SMITH, A. R.; PRYER, K. M.; SCHUETTPELZ, E.; KORALL, P.; SCHNEIDER, H.; WOLF, P. G. A classification for extant ferns. Taxon, Utrecht, v. 55, n. 3, p. 705-731, 2006.

TRYON, R. M.; TRYON A. F. Ferns and allied plants, with special reference to tropical America. New York: Springer-Verlag, 1982.

TURNER, I. M.; TAN, H. T.; CHUA, K. S. Relationships between herb layer and canopy composition in a tropical rain forest successional mosaic in Singapure. Journal of Tropical Ecology, Cambridge, v. 12, p. 843-851, 1996.

VELOSO, H. P.; RANGEL-FILHO, A. L. R.; LIMA, I. C. A. Classificação da vegetação brasileira, adaptada a um sistema universal. Rio de Janeiro: IBGE/DERMA, 1991.

YOUNG, K. R.; LÉON, B. Pteridophyte species diversity in the Central Peruvian Amazon: importance of edaphic specialization. Brittonia, Washingtom, DC, v. 41, n. 4, p. 338-395, 1989.

ZICKEL, C. S. Fitossociologia e dinâmica do estrato herbáceo de dois fragmentos florestais do estado de São Paulo. Tese (Doutorado) - Universidade Estadual de Campinas, Campinas, 1995 